\title{
Particle Filter Approach Adapted to Catadioptric Images for Target Tracking Application
}

\author{
Jean-Charles Bazin ${ }^{1}$ \\ jcbazin@rcv.kaist.ac.kr \\ Kuk-Jin Yoon ${ }^{2}$ \\ kjyoon@gist.ac.kr \\ Inso Kweon ${ }^{1}$ \\ iskweon@ee.kaist.ac.kr \\ Cedric Demonceaux ${ }^{3}$ \\ cedric.demonceaux@u-picardie.fr \\ Pascal Vasseur ${ }^{3}$ \\ pascal.vasseur@u-picardie.fr
}

${ }^{1}$ RCV laboratory

KAIST

Daejeon, South Korea

${ }^{2}$ Computer Vision laboratory

GIST

Gwangju, South Korea

${ }^{3}$ MIS laboratory

Universite de Picardie Jules Verne

Amiens, France

\begin{abstract}
This paper addresses the problem of object tracking by particle filter in catadioptric images. Whereas a large literature exists for traditional images, only a very few methods have been developed for catadioptric vision. We present two techniques to correctly deal with the strong distortion inherent to catadioptric images. First, we propose generating and diffusing the particles in the equivalent sphere space rather than in the original 2D catadioptric image. It allows to handle the specific distance associated to the distorted image in a general framework. Second, we use an adapted neighborhood to perform template matching by histogram comparison. It permits to compare templates quickly while using an active neighborhood for space-variant windows. An additional important feature of our system is that no interpolation is performed: we directly work on the original catadioptric image. Experimental results demonstrated the validity of the proposed approach.
\end{abstract}

\section{Introduction}

Target tracking is an important task in computer vision and robotic applications. The general goal is to detect a specific target and track it along time in a sequence of images. Most of existing algorithms for target tracking are composed of three important aspects. The first one is target representation (its signature) which aims to characterize the target in terms of color or shape for example. For fast execution, color representation associated with a rectangular window centered at the object is usually preferred. The second aspect is template comparison which measures the similarity of the representation of an hypothesized template with the representation of the target. Popular similarity measurements between two rectangular windows are sum of absolute difference (SAD) and normalized cross-correlation (NCC). The 
third and final aspect is the search procedure whose goal is to define a smart method to find the template that best matches with the target in the image. The most basic search procedure consists in performing a full search in the image (i.e. at every pixel location) but it is very slow. A very popular search procedure is the particle filter which is based on theoretical justifications and is studied in this paper. Particle filter $(\mathrm{PF})$ for target tracking have been widely applied and great results can be obtained.

This paper particularly focuses on target tracking in catadioptric images. Compared to traditional vision, catadioptric vision offers a much wider field of view, which permits to acquire more information from the environment. That is why more and more robots are equipped with catadioptric camera that can see in "almost every direction". It has been shown that these cameras can sensibly improve robustness and accuracy in robotic applications [3][10]. Whereas there exists a large literature on target tracking by PF on traditional images, only a very few systems have been developed for catadioptric images. Practical issues of object tracking are related to occlusion, illumination changes and scale, for which several methods have been proposed. The goal of this paper is rather dedicated to handle strong distortions inherent to catadioptric images, but the proposed approach could also be combined with some existing methods dealing with these practical issues. The basic and popular method of PF for catadioptric vision consists in, first rectifying the catadioptric image in a panoramic image and then, "blindly" applying traditional PF on the panoramic image [10][9]. The main disadvantage is that rectification requires a large amount of computation because of interpolation and introduces noise in the image. Moreover underlying distortions still exist in the rectified image. Nowadays, it is more and more admitted that the rectangular window and template matching commonly used in traditional images are not adapted for catadioptric vision. Indeed, some recent papers proposed some new neighborhood definitions and adapted template matching. For example, [19] defines some patches in the mirror surface using some ranges of elevation and azimuth angles that play the role of the height and the width of the usual rectangular shape. Then this small patch is projected in the catadioptric image. Since the size of the matching window depend on its location, the windows have different sizes. Thus for comparison, the authors normalized to a common shape and size, which enables direct measure of traditional similarity measure (e.g. $\mathrm{SAD}$ or NCC). This normalization process involves interpolation for each tested template, which considerably increases the execution time. [6] proposed a similar neighborhood definition, with the difference that the elevation and azimuth ranges are applied in the equivalent sphere surface (cf section 2) rather than the mirror surface. [17] extended the NCC method for catadioptric images where the correlation is computed on the sphere surface. This is a very interesting approach but requires large computation because of both interpolation in the sphere and computation of the NCC itself. Moreover NCC similarity measure is not really adapted for stochastic search because even if a small part of the target is not included in the template, the NCC will give a bad score. [21] proposed a particle filter-based approach for 3D tracking. This method can, in a sense, predict the distortion because it tracks the object directly in 3D. It avoids the resolution problem by using a special sensor that provides a constant resolution view of the ground plane. It leads to interesting results but requires to known apriori the shape of the object because it tries to detect/track the object boundary. Despite not directly related to catadioptric vision, we can also refer to the method of [13] developped for lens distortion. Using a specific warping matrix, the authors aimed to handle changes in perspective and scale but also the slight variations in lens distortion across the image.

Our paper is divided into four main parts. First, catadioptric projection and the equivalent sphere theorem are presented. Then, we remind particle filter algorithm for self-readability 
of the paper and clearer explanations. In the third part, we present our proposed approach for target tracking by particle filter in catadioptric images. Finally, some experiments are performed to evaluate the proposed method.

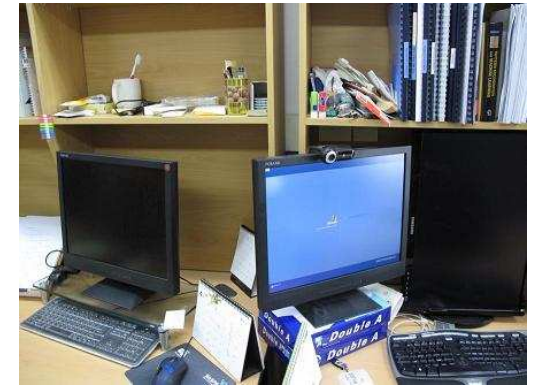

(a)

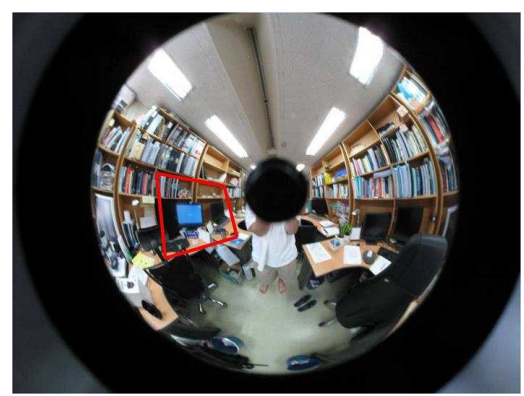

(b)

Figure 1: Compared to a traditional perspective camera (left), a catadioptric system (right) can gather much more information from the environment thanks to its wide field of view. These two images have been acquired at the same position. The red box (in the right image) corresponds to the portion of the perspective image included in the catadioptric image. In the following, we will refer to the blind spot at the image center as the "inner circle" and the large circular boundary as the "outer" circle.

\section{Catadioptric Projection}

Catadioptric cameras are a specific kind of omnidirectional sensors. They are composed of a mirror with a specific shape and a camera (perspective or telecentric). Compared to traditional cameras, they permit to acquire a much wider part of the environment (cf. Fig. 1). Baker and Nayar classified catadioptric sensors into two categories depending on the number of viewpoints [1]. Geyer and Daniilidis [11] have demonstrated the equivalence for the single viewpoint category with a two-step projection via a unitary sphere centered on the focus of the mirror (the single viewpoint). In order to apply the equivalence, it is necessary to know the intrinsic parameters of the camera and two additional parameters that define the shape of the mirror and can be estimated by calibration (refer to [11] for further details on their signification).

\section{Particle Filter for Traditional Images}

This section first briefly introduces the particle filter for target tracking in traditional images and then, presents the histogram matching technique that is used to represent and compare the object to track with an hypothesized template.

\section{Particle filter}

This section briefly presents particle filter algorithm and its associated methods such as resampling. Interested readers are invited to refer to [15] and [7] for detailed information and theoretical justifications. Particle filter (also known as condensation algorithm or boostrap 
filtering) is a widely used stochastic algorithm for approximating aposteriori density. Let $x_{t}$ be a state vector of the target at time step $t$ and $z_{t}$ be the measurement vector at the same time step $t$ with observation history $Z_{t}=\left\{z_{0}, z_{1}, \ldots, z_{t}\right\}$. The goal is to estimate the location of the target from the image measurements, i.e. the aposteriori distribution $p\left(x_{t} \mid Z_{t}\right)$. Let $s_{t}=\left\{x_{t}^{1}, \ldots, x_{t}^{N}\right\}$ be a set of $N$ particles at time $t$. A sample $x_{t}^{j}$ is selected from the set $s$ with the probability $\pi_{t}^{j}=p\left(z_{t} \mid x_{t}^{j}\right)$, where $p\left(z_{t} \mid x_{t}^{j}\right)$ is the normalized weight associated to the $j^{t h}$ particle and corresponds to the likelihood that $x_{t}^{j}$ is the true location of the target. The next section will explain how to compute this weight by histogram technique. In practice, the samples are selected using a uniform generator on $[0,1]$ and getting the corresponding sample from the cumulative distribution of $p\left(z_{t} \mid x_{t}\right)$. Then the selected samples are diffused by a dynamic model $p\left(x_{t} \mid x_{t-1}\right)$ to generate a new set of samples. For object tracking in an image sequence, a constant velocity model is usually applied for the dynamic model.

In practice, a degeneracy phenomenon might occur, i.e. all but one particle might have negligible weight after a certain number of recursive steps. Whenever a significant degeneracy is observed, a common technique is to perform a resampling step that is also designed to handle sample impoverishment (the particles that have high weights are statistically selected many times). To maintain the diversity among the particles, regularization and MCMC-move steps are two methods that are commonly applied [15]. These two methods jitter the resampled values using a Gaussian distribution whose variance is empirically computed from the weighted particles.

\section{Histogram representation and matching}

If the object to track exists in the image with no transformation compared to its representation, then pixelwise methods such as SAD or NCC will indicate a single peak value for the correct location of the target. However if the target is compared to a template that is slightly translated/rotated with respect to the true location/rotation of the target, then the similarity will be very low. As a consequence, whereas a particle may be very closed to the true location of the target, it might receive a low score. That is why these pixelwise methods usually require scanning the whole search space and are not recommended for stochastic search such as particle filter. Histogram technique presents an interesting and popular alternative for both template representation and comparison. It is slightly invariant to translation, rotation, skew and scale [14][18], and thus leads to great results for target tracking by particle filter [22]. A common way to represent a template by an histogram is based on the chromaticity space (eq 1) because it reduces the illumination change caused by the brightness variation.

$$
[r, g]=\left[\frac{R}{R+G+B} \frac{G}{R+G+B}\right]
$$

Several distances have been defined to compute the similarity between two histograms (cf [16] for a review). For experiments, we have implemented the popular Minkowski-form distance of order 1 ([20]):

$$
d\left(H_{o}, H_{t}\right)=\sum_{i=1}^{N b} \sum_{j=1}^{N b}\left|H_{o}(i, j)-H_{t}(i, j)\right|
$$

where $H_{o}$ and $H_{t}$ are the histograms associated to the object to track and the current template, and are composed of $\mathrm{Nb}$ bins for the components $r$ and $g$ of the chromaticity space. Other distances like histogram intersection, KL-distance or Bhattacharyya coefficient could also be used. 


\section{Proposed Particle Filter for Catadioptric Images}

First, this section discusses the limitations of the traditional particle filter for catadioptric images with respect to the 3 aspects listed above (representation, comparison and search). Then our proposed approach for extending particle filter to catadioptric vision is presented.

\subsection{Limitations of Traditional Particle Filter}

The distortions inherent to catadioptric images induces some important consequences for the target tracking approach presented in the previous section (particle filter associated with histogram technique). First of all, the particles should respect the distortion. For example, at the initialization, the particles are usually uniformly distributed, but intuitively, there should be more points at the center of the catadioptric image than in the boundaries. It means the sample distribution must be space-variant in the 2D image. Moreover, the variance should be appropriately updated with respect to the distortion since the distance is not linear. Concerning the histogram technique, it has been shown that the neighborhood must be space-variant: larger in the image boundary than in the center. Moreover, since neighborhoods of different size are compared, histogram matching must be extended to compare histograms of different size. We will show that working on the equivalent sphere provides a nice general framework to handle most of these aspects.

\subsection{Modifications for the Particle Filter}

We propose two important modifications for the particle filter: representation of a particle state and variance computation.

\section{Particle state}

In traditional images, particles usually take place in the $(x, y)$ space (i.e. in the image) and are manipulated in this space. In order to deal with distortions, we suggest working in the equivalent sphere space, rather than in the image 2D plane, and thus the particles should belong to the surface of the sphere. This surface can be represented using spherical angles: the azimuth $\theta \in[-\pi ; \pi]$ and the elevation $\phi \in[-\pi / 2 ; \pi / 2]$. Therefore uniformly sampling the $(\theta, \phi)$ space permits to uniformly distribute some particles while simply taking into account the distortion. Similarly, diffusing the particles is performed by simply manipulating $\theta$ and $\phi$. One may note that, in practice, $\phi$ angle is bounded by the vertical field of view of the catadioptric system: $\phi \in\left[\phi_{\text {down }} ; \phi_{u p}\right]$.

\section{Variance computation}

As explained in section 3, variance plays a key role in the regularization and MCMC-move steps to maintain the particle diversity. When the state of the particles are represented by $(x, y)$ in cartesian space, the variance can be easily computed [7]. A difficulty arises when calculating the mean and the variance of angles because of the cycling definition of angles. For example, the arithmetic mean of two angles $-160^{\circ}$ and $+160^{\circ}$ is 0 , which does not correspond to the intuitive mean angle $-180^{\circ}$ (or $180^{\circ}$ ). Thus it is clear that mean and variance of spherical angles cannot be calculated by traditional arithmetic formulae.

Let $\{\theta\}=\left\{-\pi \leq \theta_{i} \leq \pi, i=1, \ldots, N\right\}$ and $\{\phi\}=\left\{-\pi / 2 \leq \phi_{i} \leq \pi / 2, i=1, \ldots, N\right\}$ be the sets of the azimuth and elevation angles and $W=\left\{w_{i}, i=1, \ldots, N\right\}$ the set of weights assigned to each sample, computed by histogram matching in our specific application. Let 
note $\left(\bar{\theta}, \sigma_{\theta}\right)$ and $\left(\bar{\phi}, \sigma_{\phi}\right)$ the mean/variance of the sets $\{\theta\}$ and $\{\phi\}$. Circular statistics [8][4] define the mean and variance of circular quantities as follows:

$$
\begin{gathered}
\bar{\theta}=\tan ^{-1}\left(y_{\theta}, x_{\theta}\right) \text { and } \sigma_{\theta}=1-\sqrt{x_{\theta}^{2}+y_{\theta}^{2}} \\
\text { where } x_{\theta}=\sum_{i}^{N} w_{i} \cos \theta_{i} \text { and } y_{\theta}=\sum_{i}^{N} w_{i} \sin \theta_{i}
\end{gathered}
$$

By applying the same procedure for set $\{\phi\}$, we can also compute the mean and variance for the elevation angle $\phi$.

\subsection{Modifications for the Histogram Technique}

This section presents how to define space-variant neighborhood and how to compare histograms of different sizes.

\section{Neighborhood definition}

The neighborhood of a given point for perspective images is usually simply defined as the square region centered at this point. Due to distortions, such a definition is not appropriate for catadioptric images. Among the neighborhood definitions adapted to catadioptric vision, we selected [6] for his generality (definition on the sphere rather than on the mirror surface). Originally, the neighborhood of a point does not contain its own point, but for template definition, the center point is usually included. So we slightly modify [6] to include the center point. Let $P_{i}$ a point in the image plane $\mathbb{I}^{2}$ projected on the sphere $\mathbb{S}^{2}$ at $\Lambda\left(P_{i}\right)=P_{s}=(\theta, \phi, 1)$ where $\Lambda()$ is the projection function from the image plane $\mathbb{I}^{2}$ to the sphere surface $\mathbb{S}^{2}$. The spherical neighborhood of $P_{S}$, noted $N_{S}\left(P_{S}\right)$, is defined as:

$$
N_{s}\left(P_{s}\right)=\left\{\begin{array}{l}
P_{s}^{\prime}=\left(\theta^{\prime}, \phi^{\prime}, 1\right) \in \mathbb{S}^{2} \text { such that } \\
\min \left(\left|\phi-\phi^{\prime}\right|, \pi-\left|\phi-\phi^{\prime}\right|\right) \leq \phi_{\text {thresh }} \text { and } \\
\min \left(\left|\theta-\theta^{\prime}\right|, 2 \pi-\left|\theta-\theta^{\prime}\right|\right) \leq \theta_{\text {thresh }}
\end{array}\right.
$$

In plain English, the neighborhood $N_{s}\left(P_{S}\right)$ is the set of spherical points contained in a patch centered at $P_{S}$ and whose "lengths" along $\theta$ and $\phi$ directions are respectively $\theta_{\text {thresh }}$ and $\phi_{\text {thresh }}$. Then the neighborhood $N_{i}\left(P_{i}\right)$ of a point $P_{i}$ in the image plane $\mathbb{I}^{2}$ is defined as the pixels that lie in the projection of the spherical neighborhood of its spherical point onto the image plane:

$$
N_{i}\left(P_{i}\right)=\left\{P_{i}^{\prime} \in \mathbb{I}^{2} / \Lambda\left(P_{i}^{\prime}\right) \in N_{s}\left(\Lambda\left(P_{i}\right)\right)\right\}
$$

For notation, the subscripts of $N_{i}$ and $N_{s}$ are used to emphasize that $N_{i}$ and $N_{s}$ respectively corresponds to the neighborhoods in the image plane and the sphere space. These equations permit to nicely define an adapted patch for each particle on the sphere and build their associated histogram. Fig 2 shows some examples of patches obtained by this adapted neighborhood. An important remark is that no interpolation is required for this proposed neighborhood since only the pixels of the original catadioptric image are used.

\section{Histogram matching}

As the neighborhood size depends on the location of the particle, we must be able to compare histograms containing a different number of pixels. Obviously, this problem can be solved by normalizing the counts in the histogram, which can be done in two ways (cf eq 6). The basic 


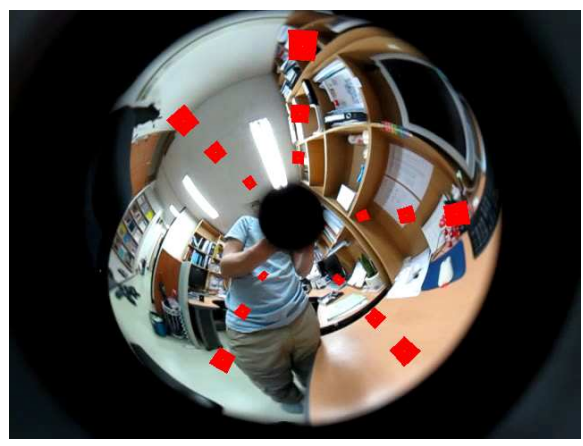

Figure 2: Example of adapted neighborhood with $\theta_{t}=\phi_{t}=4^{\circ}$. Notice the size and the shape of the patches with respect to its location.

and intuitive solution is to divide the number of observation of each bin by the total number of observations. Thus each bin corresponds to the proportion of data and their sum is 1. Another solution consists in dividing each bin by the total number of pixels and multiplying by the bin width. Whereas this normalization is less intuitive, it permits to model the distribution by a probability density function (the area under the histogram is equal to 1) [5].

$$
\bar{H}(n)=\frac{H(n)}{\sum_{i=1}^{N b} H(i)} \text { and } \bar{H}(n)=\frac{H(n) w(n)}{\sum_{i=1}^{N b} H(i)}
$$

where $H(i)$ represents the number of observations in the $i^{\text {th }}$ bin of the histogram $H$ and $w(i)$ the width of the $i^{\text {th }}$ bin.

\section{Experimental Results}

The experiments are conducted to compare two approaches for object tracking: (1) traditional particle filter in the rectified panoramic image and (2) proposed particle filter in sphere space. Our catadioptric system used in the experiments is composed of a Canon PowerShot G10 camera and a mirror from the 0-360 company, and was calibrated by [2]. We acquired 6 sequences of catadioptric images with a resolution of $640 \times 480$ at 10 frames per second. Each sequence is composed of about 1000 frames. At the first frame of the sequence, the user is invited to, first, select the object to track by clicking on the image and then, set the template size. This template is referred as "panoramic template" in the following. Then we compute the neighborhood adapted for catadioptric images (cf section 4.3). Since the template size might influence the tracking results, the neighborhood parameters $\theta_{t}$ and $\phi_{t}$ are calculated such that the number of pixels in the rectangular and adapted windows are similar for fair comparison of tracking algorithms. The template defined by the adapted neighborhood is referred as "catadioptric template" in the following. Then the histograms representing the panoramic and catadioptric templates are built (cf section 3).

For traditional PF, we rectified each catadioptric image into a panoramic image [12][9]. Rectification is composed of two steps: coordinate warping and interpolation. The coordi- 
nate transformation can be performed once in pre-processing and stored in a look-up table. As previously explained, an important disadvantage of rectification is its computation requirements due to interpolation. Moreover another practical difficulty is that the object to track might be "split" during the rectification. For example, a similar problem occurs when the world map sphere is projected into a Europe-centered panoramic map: the Pacific Ocean in split into 2 parts. In such case, the object obviously cannot be tracked properly. For fair comparison, we stored 2 look-up tables for warping and alternated between them, depending on whether or not the tracked object location in the previous frame is near the "splitting axis". For proposed PF, we simply projected the catadioptric image onto the equivalent sphere. This projection does not require any interpolation and can be done instantaneously by a look-up table computed only once in pre-processing. Due to space limitation, examples of panoramic images and spherical projections are not included.

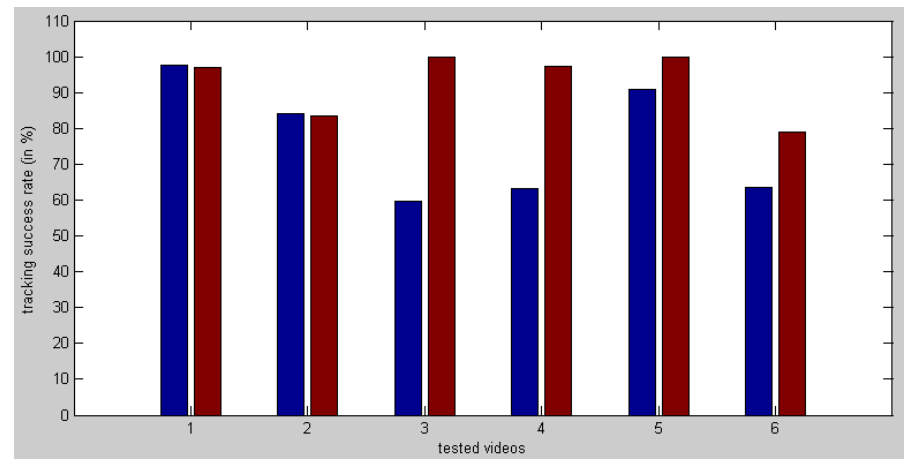

Figure 3: Comparison of tracking accuracy between traditional particle filter on rectified panoramic images (blue bars) and our proposed particle filter on original catadioptric images (red bars). Refer to the text for the definition of tracking accuracy.

The goal is to track the template in the image sequence. A track is defined as a success when the distance between the estimated location (obtained by the best particle) and the ground truth position (obtained manually) is less than a threshold (10 pixels in our experiments). Figure 3 compares the tracking accuracy obtained by traditional PF in the rectified panoramic image and the proposed PF in the original catadioptric image in the tested sequences. For the sequences 1 and 2, the traditional and proposed methods obtain similar results. By analyzing the results, we have found two explanations. First, the targets (red cup with white background and red car in parking lot) have a very specific signature for the environment of these two sequences and thus the particles easily converged to the true target location. Second, when the user selected the targets at the first frame, they were lying at about the middle distance between the inner and outer circles of the catadioptric image and thus the distortion amplitude of the target was not very high (half of the maximum distortion amplitude). In the 4 other sequences, the objects to track (car, face, magazine, phone) did not have a very specific signature. Moreover they were selected near the outer circle in the first frame and approached the image center several times during the sequence in order to demonstrate the consequences of distortion. For these sequences, the proposed method provides an higher tracking success rate. It clearly demonstrates our approach can efficiently 
handles strong distortions while performing no interpolation (for neither image rectification nor template matching) and not having to consider the "splitting" difficulty.
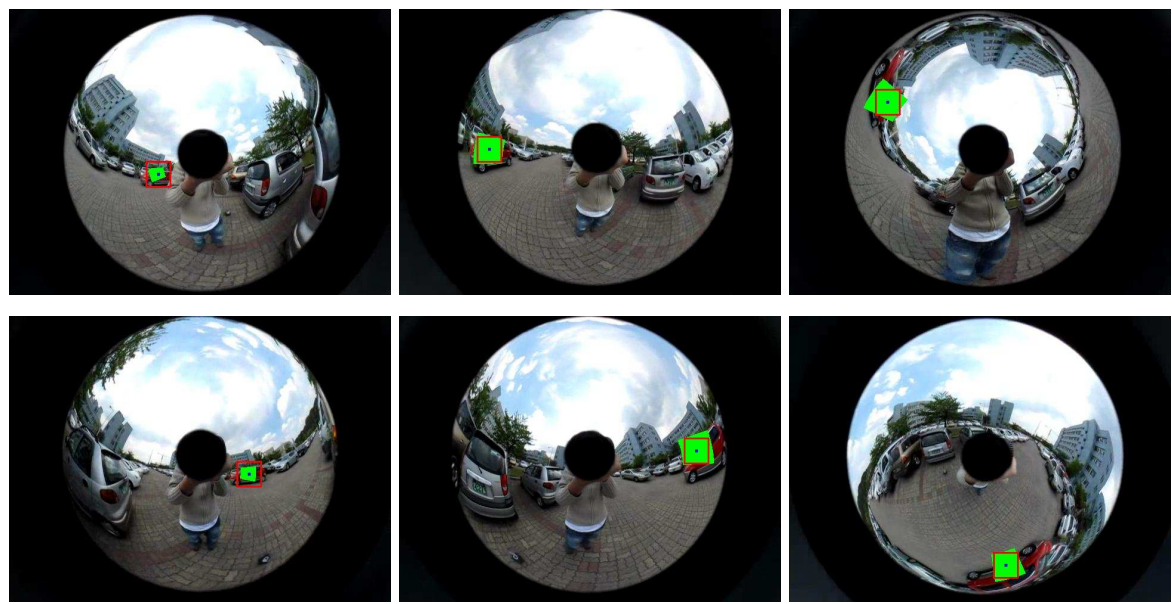

(a)

(b)

(c)

Figure 4: Examples of occlusion-reappearance (O-R) cases. Column (a): O-R by the blind spot of the inner circle and the camera holder after a yaw rotation; column (b): O-R by the field of view (outer circle) after a yaw rotation; column (c): O-R by the field of view (outer circle) after a pitch rotation.

The probability that the target is observable (i.e. lies inside the field of view) is much higher for catadioptric systems than for traditional cameras thanks to their wider field of view. Fig 4 shows some practical cases where the target gets occluded and reappears. In traditional vision, if the target leaves the field of view, it could reappear anywhere near the image boundaries. In catadioptric vision, if the object leaves the field of view by the outer (respectively inner) circle, it will likely reappear near the outer (respectively inner) circle. A simple method to consider the target "occluded" is a threshold on the histogram matching, and more advanced method could be used. If the occlusion occurs near the outer (respectively inner) circle, then we generate a new set of particles near the outer (respectively inner) circle. Examples of re-detection by the proposed approach are shown in Fig 4. A similar approach could be applied in the rectified panoramic image, but since the target location is not known, then the rectification might split the target, as discussed above. A solution would be to test the 2 versions of the panoramic views (using the 2 look-up tables) but would execute twice slower. On the contrary, our approach works directly on the original catadioptric image and thus implicitly solves this difficulty.

\section{Conclusion}

This paper addresses the problem of object tracking by particle filter in catadioptric images. The difficulty of catadioptric vision is the distortion induced by the mirror, which complicates the image processing. The proposed approach is composed of two techniques to correctly handle this important distortion. First, we proposed manipulating the particles in the equivalent sphere space rather than in the original 2D catadioptric image. It provides 
a general framework to easily handle the specific distance associated to the distorted image. Second, we combined an adapted neighborhood with histogram technique. It allows to compare templates quickly while using an active neighborhood for space-variant windows. The proposed approach provides 3 main advantages. First, it permits to correctly handle distortion and track object more robustly, as demonstrated by the experimental results. Second, no interpolation is performed (for neither image rectification nor template matching): we directly work on the original catadioptric image which leads to a faster execution. Finally, it implicitly solves the problem where the target is split during the rectification.

\section{Acknowledgments}

This work has been performed within (1) the STAR project of the Hubert Curien (Egide) partnership between RCV Lab at KAIST-Korea and MIS at UPJV-France and (2) the Korean National Strategic R\&D Program for Industrial Technology. The authors also thank Jungho Kim from RCV Lab for valuable discussions.

\section{References}

[1] S. Baker and S. K. Nayar. A theory of catadioptric image formation. In Proceedings of the International Conference on Computer Vision (ICCV’98), pages 35-42, 1998.

[2] J. P. Barreto and H. Araujo. Geometric properties of central catadioptric line images and their application in calibration. IEEE Transactions on Pattern Analysis and Machine Intelligence (PAMI’05), 27(8):1327-1333, 2005.

[3] J. C. Bazin, I.S. Kweon, C. Demonceaux, and P. Vasseur. UAV attitude estimation by vanishing points in catadioptric image. In IEEE International Conference on Robotics and Automation (ICRA'08), 2008.

[4] Christopher M. Bishop. Pattern Recognition and Machine Learning (Information Science and Statistics). Springer-Verlag New York, Inc., 2006.

[5] C. Croarkin and P. Tobias. NIST/SEMATECH e-Handbook of Statistical Methods. 2009.

[6] C. Demonceaux and P. Vasseur. Markov random fields for catadioptric image processing. Pattern Recognition Letters, 27(16):1957-1967, 2006.

[7] A. Doucet, N. De Freitas, and N. Gordon. Sequential Monte Carlo Methods in Practice. Springer-Verlag, 2001.

[8] Nicholas I. Fisher. Statistical Analysis of Circular Data. Cambridge University Press, 1995.

[9] T. Gandhi and M. M. Trivedi. Vehicle surround capture: Survey of techniques and a novel omni-video-based approach for dynamic panoramic surround maps. IEEE Transactions on Intelligent Transportation Systems (ITS'06), 7(3):293-308, 2006.

[10] J. Gaspar, N. Winters, and J. Santos-victor. Vision-based navigation and environmental representations with an omnidirectional camera. IEEE Transactions on Robotics and Automation, 16:890-898, 2000. 
[11] C. Geyer and K. Daniilidis. Catadioptric projective geometry. International Journal of Computer Vision (IJCV'01), 45(3):223-243, 2001. ISSN 0920-5691.

[12] V. Grassi and J. Okamoto. Development of an omnidirectional vision system. In Journal of the Brazilian Society of Mechanical Sciences and Engineering, volume 28, pages 58-68, 2006.

[13] G. Klein and D. Murray. Parallel tracking and mapping for small AR workspaces. In International Symposium on Mixed and Augmented Reality (ISMAR'07), Nara, Japan, November 2007.

[14] W. Niblack, R. Barber, W. Equitz, M. Flickner, E. Glasman, D. Petkovic, P. Yanker, and C. Faloutsos. The QBIC project: querying images by content using color, texture and shape. In Storage and Retrieval for Image and Video Databases (SPIE), pages 173-187, 1993.

[15] Branko Ristic, Sanjeev Arulampalam, and Neil Gordon. Beyond the Kalman Filter. Artech House, London, 2004.

[16] Y. Rubner, C. Tomasi, and L. J. Guibas. The earth mover's distance as a metric for image retrieval. International Journal of Computer Vision (IJCV'00), 40(2):99-121, 2000.

[17] L. Sorgi and K. Daniilidis. Normalized cross-correlation for spherical images. In Proceedings of the European Conference on Computer Vision (ECCV'04), volume 3022, pages 542-553, 2004.

[18] M. A. Stricker and M. Orengo. Similarity of color images. In Storage and Retrieval for Image and Video Databases (SPIE), pages 381-392, 1995.

[19] T. Svoboda and T. Pajdla. Matching in catadioptric images with appropriate windows, and outliers removal. In International Conference on Computer Analysis of Images and Patterns (CAIP '01), pages 733-740, 2001.

[20] M.J. Swain and D.H. Ballard. Color indexing. International Journal of Computer Vision (IJCV'91), 7(1):11-32, 1991.

[21] M. Taiana, J. Gaspar, J. Nascimento, A. Bernardino, and P. Lima. 3d tracking by catadioptric vision based on particle filters. pages 77-88, 2008.

[22] K.J. Yoon, G.J. Jang, S.H. Kim, and I.S. Kweon. Fast landmark tracking and localization algorithm for mobile self-localization. In IFAC Workshop on Mobile Robot Technology, pages 190-195, 2001. 